Frayzer Lima de Almeida iD frayzerlima@ufpa.br Universidade Federal do Pará (UFPA)

\section{Modelagem epidemiológica determinística a partir de dados reais da Covid-19 no estado do Pará entre os meses de março e abril de 2020}

\section{Deterministic epidemiological modeling on real data from Covid-19 in the state of Pará between March and April 2020}
Modelado epidemiológico determinístico desde datos reales de Covid-19 en el estado del Pará entre los meses de marzo $y$ abril de 2020

\section{Modélisation épidémiologique déterministe de données réelles du Covid-19 dans i'état de Pará au Brésil entre mars et avril 2020}

\section{Resumo}

Neste trabalho, aplicamos o modelo epidemiológico determinístico SIR clássico (suscetíveis, infectados, recuperados) sem dinâmica vital, o qual foi modelado e solucionado pelo método numérico de Runge-Kutta de $3^{\text {a }}$ ordem, sobre dados reais da Covid-19 no estado do Pará entre os meses março e abril de 2020. Para realizar as simulações computacionais, incluímos o método de Runge-Kutta no ambiente Octave, assim como o modelo determinístico SIR. Os parâmetros do modelo foram determinados a partir dos dados reais da Covid-19. As soluções numéricas dos infectados aproximaram-se aos dados reais, os quais foram adquiridos no site da Secretaria de Saúde do Estado do Pará (Sespa), validando a metodologia acima proposta. A partir das soluções numéricas, foi possível compreender a dinâmica da Covid-19 no estado do Pará. Constatamos que a curva de infectados possui crescimento entre 04 de maio e 08 de junho de 2020, e atinge seu ponto máximo em 11 de junho de 2020, quando a curva de infectados decresce, sendo assintótica para zero a partir do dia 20 de julho de 2020.

Palavras-chave: Epidemiologia. Covid-19. SIR. Runge-Kutta.

\begin{abstract}
In this work we apply the deterministic epidemiological model, classic SIR (susceptible, infected, recovered) without vital dynamics, on real data from Covid-19 in the State of Pará between March and April 2020, which was modeled and solved by the numerical method 3rd order Runge-Kutta. To perform computer simulations, we include the Runge-Kutta method in the OCTAVE environment, as well as the deterministic SIR model. The parameters of the deterministic SIR model were determined from the aforementioned real data from Covid-19. The numerical solutions of those infected came close to the actual data, which were acquired on the website of the State Health Department of Pará (SESPA), thus validating the methodology proposed above. From the numerical solutions it was possible to understand the dynamics of Covid-19 in the State of Pará. We found that the infected curve has a growth between May 4 and June 82020, and reaches its peak on June 11,2020, from that date the infected curve decreases, being asymptotic to zero from the 20th July 2020.
\end{abstract}

Keywords: Epidemiology. Covid-19. SIR, Runge-Kutta. 


\begin{abstract}
Resume
En este trabajo empleamos el modelo epidemiológico determinístico, SIR clásico (susceptibles, infectados, recuperados) sin dinámica vital, sobre datos reales de Covid-19 en el Estado del Pará entre los meses de marzo y abril de 2020, lo cual fue moldeado y resuelto por el método numérico de Runge-Kutta de $3^{\circ}$ orden. Para realizar las simulaciones computacionales, agregamos el método de Runge-Kutta en el ambiente OCTAVE, y también el modelo determinístico SIR. Los parámetros del modelo determinístico SIR fueron determinados a partir de los datos reales de Covid-19. Las soluciones numéricas de los infectados se acercaron a los datos reales, los cuales fueron obtenidos en la página web de la Secretaria de Salud del Estado del Pará (SESPA), validando la metodología aquí propuesta. A partir de las soluciones numéricas fue posible comprender la dinámica de Covid-19 en el Estado del Pará. Constatamos que la curva de infectados posee crecimiento entre 04 de mayo y 08 de junio de 2020, y alcanza su punto máximo en 11 de junio de 2020. A partir de esta fecha la curva de infectados decrece, convirtiéndose en asintótica para cero a partir del día 20 de julio de 2020.
\end{abstract}

Palabras-clave: Epidemiología. Covid-19. SIR. Runge-Kutta.

\section{Résumé}

Dans ce travail, on a appliqué le modèle épidémiologique déterministe, le SIR (sensible, infecté, récupéré) sans dynamique vitale, sur des données réelles du Covid-19 dans l'État du Pará, au Brésil entre mars et avril 2020, qui a été modélisé et résolu par la méthode numérique Runge-Kutta de 3 à ordre. Pour effectuer des simulations computationnelles, on a inclus la méthode Runge-Kutta et le modèle déterministe SIR dans l'environnement Octave. Les paramètres du modèle déterministe SIR ont été déterminés à partir des données réelles susmentionnées du Covid-19. Les solutions numériques des infectées ont été proche des données réelles. Celles-là ont été acquises sur le site Web du Département de la Santé de l'État du Pará (SESPA), ce qui valide la méthodologie proposée ci-dessus. À partir des solutions numériques, il a été possible de comprendre la dynamique du Covid-19 dans l'État du Pará. On a pu constater que la courbe des infectés a une croissance entre le 4 mai et le 8 juin 2020, et atteint son point maximum le 11 juin 2020. À partir de cette date, la courbe des infectée diminue, en étant asymptotique à zéro à partir du 20 juillet 2020.

Mots-clés: Épidémiologie. Covid-19. SIR. Runge-kutta.

\section{Introdução}

Coronavírus é uma família de vírus que causam infecções respiratórias (MS, 2020). Foram isolados pela primeira vez em 1937, no entanto foi em 1965 que foi descrito como coronavírus, em decorrência do perfil na microscopia, parecendo uma coroa. Os coronavírus mais comuns que infectam humanos são o alpha coronavírus 229E e NL63 e beta coronavírus OC43 e HKU1 (FCV, 2020). Até o momento, os tipos de coronavírus conhecidos são: Alpha coronavírus 229E e NL63, Beta coronavírus OC43 e HKU1, SARS-CoV (causador da Síndrome Respiratória do Oriente Médio ou MERS) e o SARS-CoV-2, novo tipo de vírus do agente coronavírus, chamado de novo coronavírus (FCV, 2020). Este último agente do coronavírus foi descoberto em dezembro de 2019, após casos registrados na China (MS, 2020). A transmissão dos coronavírus costuma ocorrer pelo ar ou por contato pessoal com secreções contaminadas, como gotículas de saliva proveninentes de espirro e tosse, catarro no contato pessoal, como toque ou aperto de mão, e com objetos ou superfícies contaminadas, seguido de contato com a boca, nariz ou olhos (FCV, 2020). A partir da infecção por coronavírus, o seu período de incubação é de 5 dias, com intervalos que podem chegar a 12 dias, período que os primeiros sintomas demoram para aparecer (FCV, 2020). Os sintomas da Covid-19 podem variar de um simples resfriado até 
uma pneumonia severa, sendo os sintomas mais comuns tosse, febre, coriza, dor de garganta e dificuldade para respirar (MS, 2020).

Segundo o Ministério da Saúde (2020), em 14 de abril, o Brasil apresentou 23.430 casos confirmados e 1.328 óbitos por Covid-19, com um percentual de letalidade de 5,7\%. O Pará, em 14 de abril, apresentou 270 casos confirmados, com 15 óbitos por Covid-19, uma letalidade de 5,6\%. Devido ao compartilhamento da Secretaria de Saúde do Estado do Pará (Sespa), por meio de seu site, de dados reais acerca dos casos registrados da Covid-19 no Pará, é possível desenvolver pesquisas sobre a dinâmica da Covid-19 nesse estado, proporcionando, por parte de entidades públicas, uma tomada de decisão para combater essa pandemia mundial.

Dessa forma, a partir de dados reais da Covid-19 compartilhados pelo site da Sespa entre os meses março e abril de 2020 (SESPA, 2020), o presente trabalho propõe, utilizando o modelo epidemiológico determinístico SIR (suscetíveis, infectados, recuperados), modelar e solucionar esse modelo epidemiológico determinístico através do método numérico de Runge-Kutta de $3^{\mathrm{a}}$ ordem, estudar o comportamento e/ou a dinâmica da referida pandemia no estado do Pará e calcular suas estimativas de previsão, tanto de suscetíveis e recuperados quanto de infectados.

\section{Objetivos}

- Desenvolver modelagem através do método de Runge-Kutta de $3^{a}$ ordem sobre o modelo epidemiológico determinístico SIR sem dinâmica vital (ARAÚJO, 2015; SOARES, 2010).

- Relacionar o modelo epidemiológico determinístico SIR, por meio de seus parâmetros, aos dados reais de Covid-19, constantes no site da Sespa, entre os meses março e abril de 2020 (SESPA, 2020).

- Analisar a dinâmica, assim como a previsão da Covid-19 no estado do Pará, utilizando a solução numérica, segundo o método de Runge-Kutta de $3^{\text {a }}$ ordem, sobre dados reais da Covid-19 entre os meses de março e abril de 2020, constantes no site da Sespa (SESPA, 2020).

\section{Metodologia}

Nesta seção, apresentamos o modelo epidemiológico determinístico SIR sem dinâmica vital e a sua modelagem segundo o método de Runge-Kutta de $3^{\mathrm{a}}$ ordem. Em seguida, determinamos os parâmetros do modelo epidemiológico SIR a partir de dados reais da Covid-19 entre os meses março e abril de 2020, divulgados pelo site da Sespa (SESPA, 2020).

\subsection{O modelo epidemiológico SIR}

O modelo SIR, Fig. 1, assume que os indivíduos podem ser suscetíveis, S, infecciosos, I, ou recuperados, R. Dessa forma, os suscetíveis são aqueles que podem contrair a doença; os infecciosos são os que estão doentes e podem transmitir a doença; e os recuperados são os que contraíram a doença e adquiriram imunidade ou morreram. Na Fig. 1, $\beta$ representa a taxa de transmissão, que determina o número médio de contatos adequados entre suscetíveis e infecciosos por unidade de tempo, e $\mu$ corresponde à taxa de recuperação, isto é, ao número de indivíduos que deixam de ser infecciosos por unidade de tempo. Esses modelos são aplicados na modelagem de doenças infecciosas como rubéola, varicela e sarampo (ARAÚJO, 2015; SOARES, 2010). Como ainda não temos informações de indivíduos curados e/ou recuperados de Covid-19 que voltaram novamente a se infectar, escolhemos o referido modelo SIR, Fig. 1, para desenvolver nossas propostas metodológicas presentes neste artigo. 
Figura 1- Modelo SIR clássico.

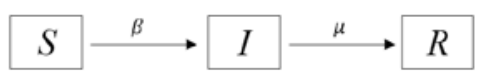

Fonte: Araújo (2015).

O modelo SIR padrão é modelado por um sistema de equações diferenciais ordinárias, o qual é chamado de modelo epidemiológico determinístico SIR. A partir dos dados, Tab. (1), aplicamos o modelo determinístico SIR para analisar a população de infectados e a de suscetíveis e recuperados no estado do Pará, assim como calcular estimativas de previsão. O modelo epidemiológico determinístico SIR está expresso, abaixo, pelo seguinte sistema de equações diferenciais ordinárias, Eq. (3.1):

$$
\begin{aligned}
& \left\{\begin{array}{l}
d S(t) / d t=-\beta S(t) I(t) \\
d I(t) / d t=\beta S(t) I(t)-\mu I(t) \\
d R(t) / d t=\mu I(t)
\end{array}\right. \\
& \text { com } \mathrm{S}\left(\mathrm{t}_{0}\right)=\mathrm{S}_{0}, \quad \mathrm{I}\left(\mathrm{t}_{0}\right)=\mathrm{I}_{0} \text { e } \mathrm{R}\left(\mathrm{t}_{0}\right)=\mathrm{R}_{0} .
\end{aligned}
$$

Em que: $\mu$ é a taxa de recuperação; $\beta$ é a taxa de transmissão da doença; $S(t)$ é a função que descreve a quantidade de indivíduos suscetíveis à Covid-19 no tempo $t \geq 0 ; I(t)$ é a função que descreve a quantidade de indivíduos infectados pela Covid-19 também no tempo $t \geq 0 ; R(t)$ é a função que descreve os indivíduos recuperados. O número total de indivíduos é dado por $N(t)=S(t)+I(t)+R(t)$, e $S_{0}, I_{0}$ e $R_{0}$ são a quantidade de indivíduos suscetíveis, infectados e recuperados, respectivamente, no tempo inicial $t_{0} \geq 0$. Consideramos a população constante, com $d N(t) / d t=0$, ou seja, $d S(t) / d t+d l(t) / d t+d R(t) / d t=0$.

\subsection{O modelo epidemiológico SIR modelado e solucionado pelo método numérico de Runge-Kutta de $3^{\mathrm{a}}$ ordem}

Consideremos o sistema de equações diferenciais ordinárias com três equações e a variável tempo implícita:

$$
\left\{\begin{array}{l}
\frac{d S(t)}{d t}=f(S(t), I(t), R(t)) \\
\frac{d I(t)}{d t}=g(S(t), I(t), R(t)) \\
\frac{d R(t)}{d t}=l(S(t), I(t), R(t))
\end{array}\right.
$$

$\operatorname{com} S\left(t_{0}\right)=S_{0}, I\left(t_{0}\right)=I_{0}$ e $R\left(t_{0}\right)=R_{0}$.

Assim, temos o problema de valor inicial, em que se assume que $f, g$ e $l$, e sejam contínuas e suas derivadas parciais de primeira e segunda ordens contínuas e limitadas na região $a \leq t \leq b,-\infty<f<+\infty,-\infty<$ g $<+\infty$ e $-\infty<\mathrm{I}<+\infty$. A partir dessas hipóteses, é possível calcular aproximações para a solução do problema de valor inicial acima, que é o que apresentamos a seguir.

Um dos métodos numéricos para resolver o problema de valor inicial, Eq. (3.2), é o método de RungeKutta de terceira ordem (BARROSO et al., 1987; SCHEID, 1989), o qual é descrito a seguir:

$$
\begin{aligned}
& \left\{\begin{array}{l}
S\left(t_{i+1}\right)=S\left(t_{i}\right)+h \varphi\left(S_{i}, I_{i}, R_{i}\right) \\
I\left(t_{i+1}\right)=I\left(t_{i}\right)+h \omega\left(S_{i}, I_{i}, R_{i}\right) \\
R\left(t_{i+1}\right)=R\left(t_{i}\right)+h \theta\left(S_{i}, I_{i}, R_{i}\right)
\end{array}\right. \\
& \text { com t }_{i+1}-t_{i}=h, i=0,1, \ldots, m-1
\end{aligned}
$$


Em que $\varphi\left(S_{i}, I_{i,} R_{i}\right)=a K_{1}+\beta K_{4}+y K_{7}, \omega\left(S_{i}, I_{i,} R_{i}\right)=a K_{2}+\beta K_{5}+y K_{8 e}, \theta\left(S_{i}, I_{i} R_{i}\right)=a K_{3}+\beta K_{6}+y K_{9}$ em que $K_{1=} f\left(S_{i}, I_{i,} R_{i}\right), K_{2}=g\left(S_{i}, I_{i,} R_{i}\right), K_{3=} \mathrm{l}\left(S_{i}, I_{i,} R_{i}\right), K_{4=} f\left(S_{i j}+p h K_{1, i} l_{i}+q h K_{2} R_{i}+r h K_{3}\right), K_{5} g f\left(S_{i}+p h K_{1,} l_{i}+q h K_{2} R_{i}+\right.$

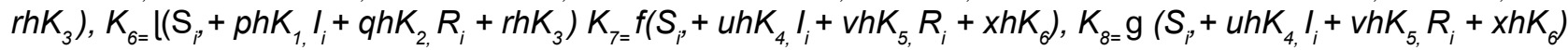

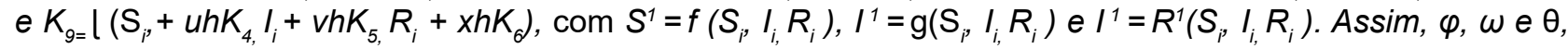
são as funções incrementos e é o comprimento do passo, sendo $S\left(t_{i+1}\right), I\left(t_{i+1}\right)$ e $R\left(t_{i+1}\right)$, respectivamente, as aproximações de suscetíveis, infectados e recuperados no tempo $t_{i+1}$, com i $=0,1, \ldots, \mathrm{m}-1$. Assim, temos $S\left(t_{i}\right)$ $=S i, I\left(t_{i}\right)=I_{i}$ e $R_{i}, I\left(R_{i}\right), \mathrm{com}=0,1, \ldots, \mathrm{m}-1$.

As constantes $a, \beta, y, p, r, u, v$ e $x$ em $\mathrm{K}_{4}, \mathrm{~K}_{5}, \mathrm{~K}_{6}, \mathrm{~K}_{7}, \mathrm{~K}_{8}$ e $\mathrm{K}_{9}$ devem ser determinadas para que se obtenha o método de Runge-Kutta de $3^{\mathrm{a}}$ ordem.

Por um lado, entendendo as funções $\mathrm{K}_{4}, \mathrm{~K}_{5}, \mathrm{~K}_{6}, \mathrm{~K}_{7}, \mathrm{~K}_{8}$ e $\mathrm{K}_{9}$ em três variáveis, desenvolvemos as expansões de $\mathrm{K}_{4}, \mathrm{~K}_{5}, \mathrm{~K}_{6}, \mathrm{~K}_{7}, \mathrm{~K}_{8}$ e $\mathrm{K}_{9}$ respectivamente, na série de Taylor até o termo de $2^{\mathrm{a}}$ ordem no ponto $\left(\mathrm{S}_{\mathrm{i}}, \mathrm{I}_{\mathrm{i}} \mathrm{R}_{\mathrm{i}}\right)$, obtendo aproximações para $\mathrm{K}_{4}, \mathrm{~K}_{5}, \mathrm{~K}_{6}, \mathrm{~K}_{7}, \mathrm{~K}_{8}$ e $\mathrm{K}_{9}$. Ao substituirmos $\mathrm{K}_{1}, \mathrm{~K}_{2}, \mathrm{~K}_{3}$ e as aproximações de $\mathrm{K}_{4}, \mathrm{~K}_{5}, \mathrm{~K}_{6}, \mathrm{~K}_{7}, \mathrm{~K}_{8}$ e $\mathrm{K}_{9}$ na Eq. (3.3), obtivemos aproximações para $S\left(t_{i+1}\right), I\left(t_{i+1}\right)$ e $R\left(t_{i+1}\right)$, segundo a série de Taylor de $3^{\mathrm{a}}$ ordem em três variáveis.

Por outro lado, expandimos, em séries de Taylor de $3^{\text {a }}$ ordem, as soluções teóricas $S(t), I(t)$ e $R(t)$ no ponto resultando em aproximações para $S\left(t_{i+1}\right), I\left(t_{i+1}\right)$ e $R\left(t_{i+1}\right)$, segundo a série de Taylor de $3^{\mathrm{a}}$ ordem em uma variável.

Agora, considerando as aproximações das soluções $S\left(t_{i+1}\right), I\left(t_{i+1}\right)$ e $R\left(t_{i+1}\right)$, obtidas segundo a série de Taylor de $3^{\mathrm{a}}$ ordem em três variáveis e segundo a série de Taylor de $3^{\mathrm{a}}$ ordem em uma variável, comparamos os termos de mesma potência, com relação à $h$, tanto para $S\left(t_{i+1}\right)$ e $I\left(t_{i+1}\right)$ quanto para $R\left(t_{i+1}\right)$ respectivamente, e obtemos o seguinte sistema não linear:

$$
\left\{\begin{array}{l}
\alpha+\beta+\gamma=1 \\
\beta p+\gamma u=1 / 2 \\
\gamma u^{2}+\beta p^{2}=1 / 3 \\
\gamma u p=1 / 6 \\
\beta p q+\gamma u v=1 / 3 \\
\gamma v p=1 / 6 \\
\gamma u q=1 / 6 \\
\gamma v^{2}+\beta q^{2}=1 / 3 \\
\gamma v q=1 / 6 \\
\beta q+\gamma v=1 / 2
\end{array}\right.
$$

Considerando que todas essas incógnitas são diferentes de zero, com com $u=v=x$ e $p=q=r$, e tomando $u=v=x=a$ e $p=q=r=b$, temos:

$$
\left\{\begin{array}{c}
\alpha+\beta+\gamma=1 \\
\beta b+\gamma a=1 / 2 \\
\gamma a^{2}+\beta b^{2}=1 / 3 \\
\gamma a b=1 / 6
\end{array}\right.
$$

Finalmente, para resolvê-lo, tomamos $a=b$ e $\beta=\gamma$, daí temos: $a=b=2 / 3, \beta=\gamma=3 / 8$ e $\alpha=1 / 4$. Dessa forma, temos que: $p=q=r=u=v=x=2 / 3, \beta=y=3 / 8$ e $\alpha=1 / 4$.

Essa modelagem, segundo o método numérico de Runge-Kutta de $3^{\text {a }}$ ordem sobre o modelo SIR, foi implementada no ambiente computacional Octave, a partir do qual obtemos as soluções aproximadas para os suscetíveis, os infectados e os recuperados no estado do Pará. No entanto, para chegarmos a esse resultado, precisamos determinar os parâmetros $\mu$ e $\beta$ do modelo SIR, Eq. (3.1), o que vamos discutir a seguir.

\subsection{A determinação dos parâmetros e do modelo SIR em relação aos dados reais da Covid-19}

Para determinar os parâmetros $\mu$ e $\beta$ do modelo determinístico SIR, Eq. (3.1), utilizamos os dados reais da Covid-19, expedidos pelo site da Sespa, entre os meses de março e abril de 2020 , os quais estão apresentados na Tab. (1) abaixo. 
Tabela 1 - Números de infectados por Covid-19 no Pará entre os meses de março e abril de 2020.

\begin{tabular}{|c|c|}
\hline Dias & $\mathrm{N}^{\circ}$ de infectados \\
\hline $18 / 03 / 20$ & 01 \\
\hline $19 / 03 / 20$ & 01 \\
\hline $20 / 03 / 20$ & 02 \\
\hline $21 / 03 / 20$ & 02 \\
\hline $22 / 03 / 20$ & 04 \\
\hline $23 / 03 / 20$ & 05 \\
\hline $24 / 03 / 20$ & 05 \\
\hline $25 / 03 / 20$ & 07 \\
\hline $26 / 03 / 20$ & 13 \\
\hline $27 / 03 / 20$ & 16 \\
\hline $28 / 03 / 20$ & 18 \\
\hline $29 / 03 / 20$ & 23 \\
\hline $30 / 03 / 20$ & 26 \\
\hline $31 / 03 / 20$ & 32 \\
\hline $01 / 04 / 20$ & 41 \\
\hline $02 / 04 / 20$ & 48 \\
\hline $03 / 04 / 20$ & 75 \\
\hline $04 / 04 / 20$ & 78 \\
\hline $05 / 04 / 20$ & 86 \\
\hline $06 / 04 / 20$ & 123 \\
\hline $07 / 04 / 20$ & 154 \\
\hline 08/04/20 & 165 \\
\hline $09 / 04 / 20$ & 169 \\
\hline $10 / 04 / 20$ & 217 \\
\hline $11 / 04 / 20$ & 237 \\
\hline $12 / 04 / 20$ & 259 \\
\hline $13 / 04 / 20$ & 310 \\
\hline
\end{tabular}

Fonte: Secretaria de Saúde do Estado do Pará (2020).

Apesar de na Tab.(1) constar o quantitativo de infectados por Covid-19 no Pará, consideramos nas simulações a população $N(t)$ igual ao total de habitantes em Belém e Ananindeua, ou seja, 1492745+530598, respectivamente (IBGE, 2020), por serem os locais com um quantitativo maior de infectados.

O modelo da Eq.(3.1) possui dois parâmetros, então, para os seus cálculos, levamos em consideração a taxa de letalidade de 5,6\%. Dessa forma, foi possível calcular uma estimativa dos recuperados e, consequentemente, os suscetíveis, pois $N(t)=S(t)+l(t)+R(t)$.

Tais parâmetros foram calculados considerando o referido modelo da Eq. (3.1) como um sistema de equações algébricas, em que suscetíveis, infectados e recuperados são as suas respectivas médias (normalizadas pelo total de habitantes de ambos os municípios), assim como as suas taxas e/ou derivadas. Para o cálculo das médias das derivadas, foram utilizadas aproximações aos dados reais de infectados, recuperados e suscetíveis (normalizados pelo total de habitantes de Belém e Ananindeua), por meio de interpolação linear e derivação numérica dos dados de infectados da Tab.(1), e as estimativas de suscetíveis e recuperados. Assim, temos um sistema linear com três equações e duas incógnitas ( $\beta$ : taxa de contato, $\mu$ : taxa de recuperação), em que obtemos: $\mu=0.14337348375320$ e $\beta=0.29528058079588$. 
Dado que esses parâmetros são determinados a partir de equações algébricas normalizadas pelo total da população de Belém e Ananindeua, cabe ressaltar que, na inclusão do método de Runge-Kutta de $3^{\mathrm{a}}$ ordem sobre o modelo determinístico SIR no ambiente Octave, Eq. (3.1), as soluções aproximadas de suscetíveis, infectados e recuperados estão normalizadas durante o processamento, porém, ao final do processo, as soluções são desnormalizadas.

\section{Resultados e discussão}

Nesta seção, vamos analisar a dinâmica da Covid-19 no estado do Pará, assim como as estimativas de previsões de suscetíveis, infectados e recuperados a partir do mês de abril de 2020.

\subsection{A dinâmica da Covid-19 no estado do Pará}

Nesta seção, vamos apresentar as soluções do modelo epidemiológico SIR, por meio do método numérico de Runge-Kutta de $3^{\text {a }}$ ordem, a partir de dados reais da Covid-19 no estado do Pará entre os meses março e abril de 2020, Tab.(1), assim como analisar a monotonicida das soluções e previsões da Covid-19 no Pará a partir do mês de abril de 2020.

Dessa forma, para o modelo SIR, Eq. (3.1), a partir das seguintes condições iniciais $S_{0}=2.023331919200000$ $\mathrm{e}+006, \mathrm{I}_{0}=5.7000$ e $\mathrm{R}_{0}=5.3808$, com os parâmetros $\beta$ e $\mu$, temos o comportamento dos infectados e recuperados na Fig. 2. No eixo horizontal da Fig. 2, o dia 18 está para 18 de março (dia 18 - 18 de março), assim por diante. Como o mês de março vai até o dia 31 , o dia 32 está para $1^{\circ}$ de abril (dia $32-1^{\circ}$ de abril), o dia 33 para 02 de abril (dia 33 - 02 de abril), e assim por diante. Por exemplo, no dia 44 (13 de abril) foram registrados 310 indivíduos infectados por Covid-19, conforme Fig. 2.

Figura 2 - Infectados no Pará entre os dias 18 de março e 13 de abril de 2020 (círculos), com as simulações de infectados (em vermelho) e recuperados (em azul) entre os dias 18 de março e 20 de abril de 2020.

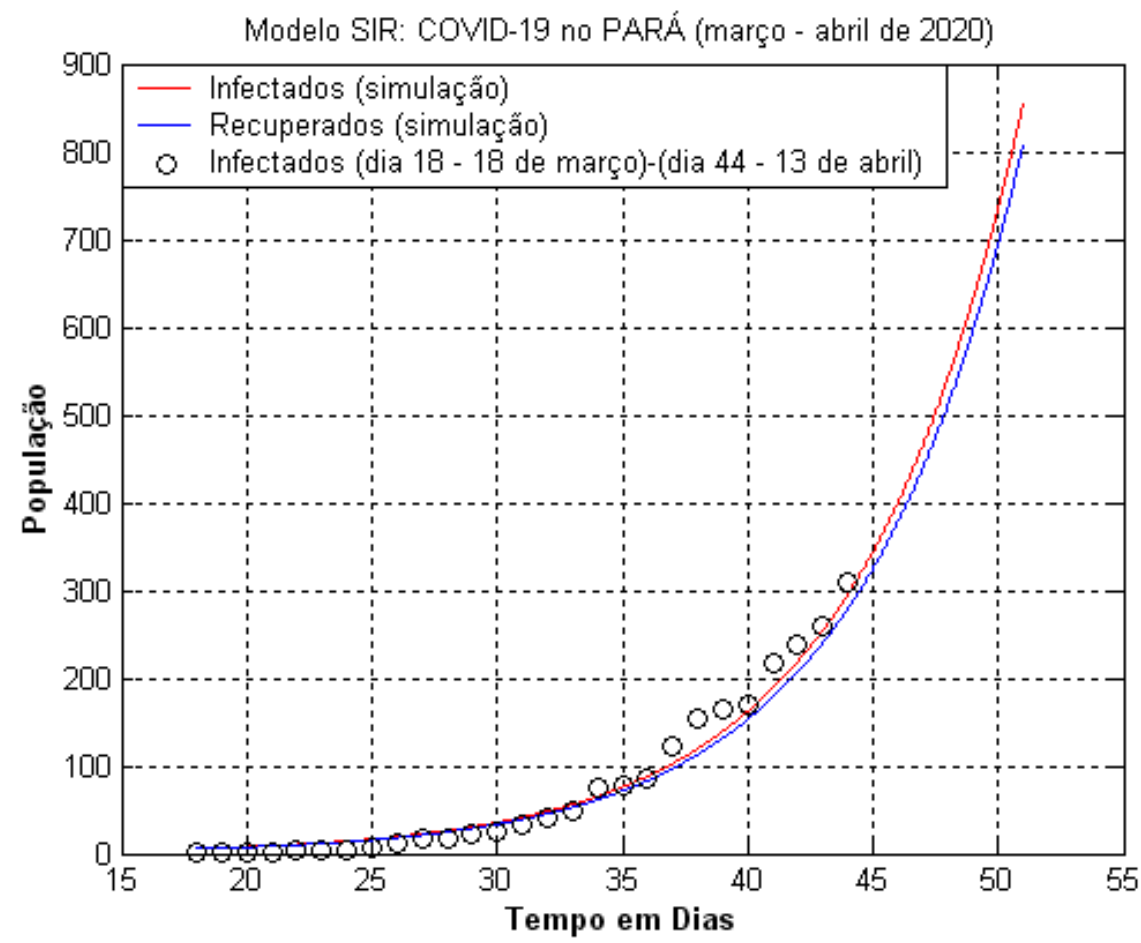

Fonte: Próprio autor, 2020. 
Observamos, na Fig. 2, que a simulação de infectados (em vermelho) aproxima-se dos dados reais de infectados (círculos) no estado do Pará. Também é verificado que a simulação de infectados cresce com o passar dos dias, sendo esse crescimento de ordem exponencial. A partir do dia 44 (13 de abril), é possível realizar estimativas de previsão através da curva de simulação de infectados (em vermelho) presente na Fig. 2. Assim, por exemplo, no dia 51 (20 de abril), o modelo prevê aproximadamente 867 indivíduos infectados no estado do Pará. Portanto, em relação ao dia 13 de abril, o modelo prevê um crescimento aproximado de infectados de $279 \%$ até o dia 20 de abril.

Agora, nas mesmas condições iniciais e com os mesmo valores dos parâmetros $\beta$ e $\mu$, temos o comportamento dos suscetíveis, infectados e recuperados, na Fig. 3, entre os dias 18 de março (dia 18) e 18 de junho (dia 110) de 2020.

Figura 3 - Simulações de infectados (em vermelho), recuperados (em azul) e suscetíveis (em preto) entre os dias 18 de março (dia 18) e 18 de junho (dia 110) de 2020.

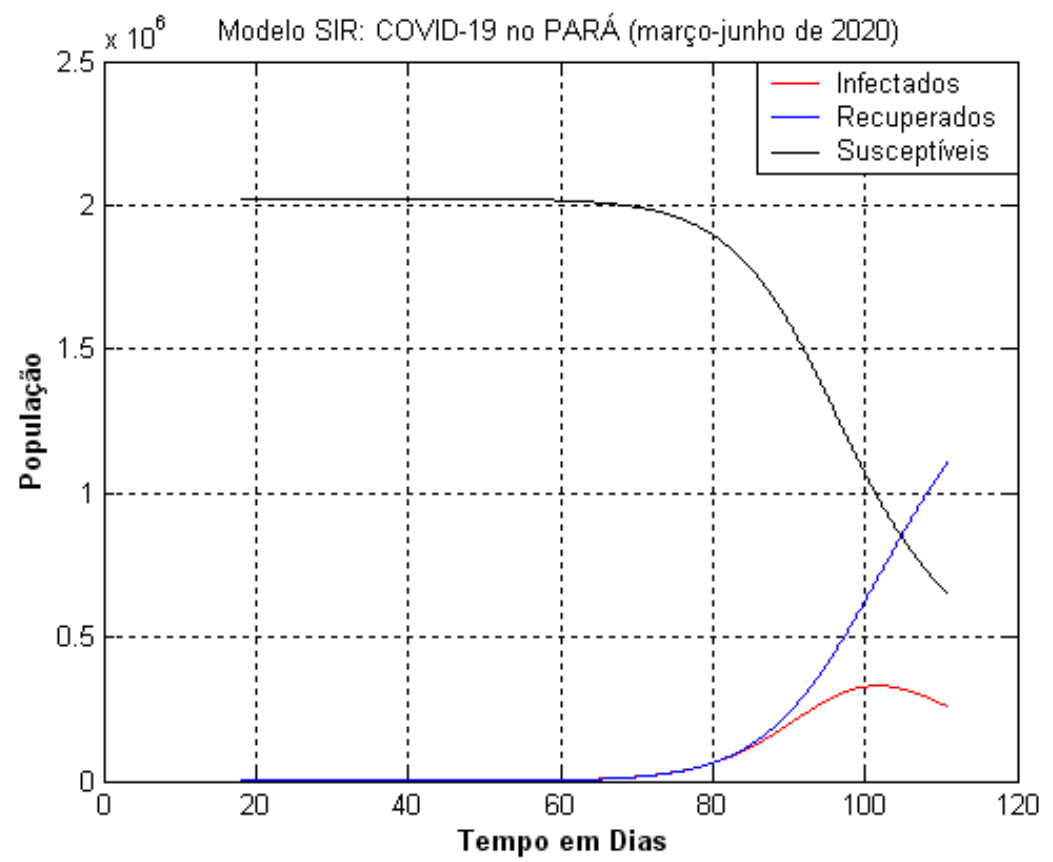

Fonte: Próprio autor, 2020.

Observamos, na Fig. 3, que entre os dias 04 de maio (dia 65) e 08 de junho (dia 100) de 2020 ocorre um acentuado crescimento dos recuperados (em azul), acompanhado por um crescimento menos acentuado dos infectados (em vermelho). Simultaneamente, nesse mesmo período, observamos um acentuado decrescimento dos suscetíveis. Além disso, observamos que a curva de infectados atinge o seu ponto máximo no dia 11 de junho (dia 103) com aproximadamente $2.9 \times 10^{5}$ indivíduos infectados no estado do Pará, o que corresponde a, aproximadamente, $11.6 \%$ dos habitantes dos municípios de Belém e Ananindeua. A partir do dia 11 de junho (dia 103) observamos um decrescimento da curva de infectados (em vermelho), conforme Fig. 3.

Ainda, sob as mesmas condições iniciais e os mesmo valores dos parâmetros $\beta$ e $\mu$, temos o comportamento dos suscetíveis, infectados e recuperados na Fig. 4 entre os dias: 18 de março (dia 18) e 20 de julho (dia 142) de 2020. 
Figura 4 - Simulações de infectados (em vermelho), recuperados (em azul) e suscetíveis (em preto) entre os dias 18 de março (dia 18) e 20 de julho (dia 142) de 2020.

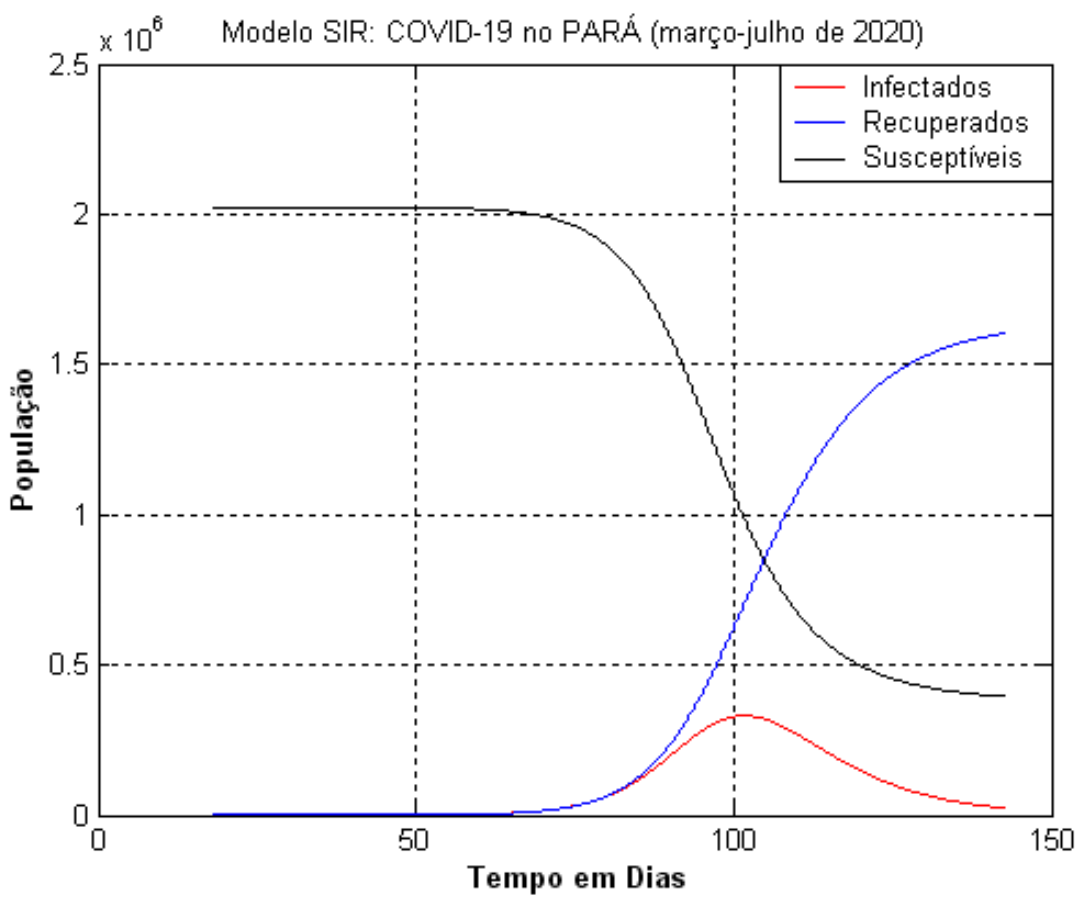

Fonte: Próprio autor, 2020

Finalmente, na Fig. 4 observamos que, a partir do 20 de julho (dia 142), existe uma tendência para estabilização dos infectados (em vermelho), recuperados (em azul) e suscetíveis (em preto). Uma vez que é possível constatar que os suscetíveis tendem para, aproximadamente, $16.8 \%$ da população, os infectados tendem, assintoticamente, para zero, ou seja, a doença tende a extinguir-se. Também observamos que existe uma tendência nos recuperados, Fig. 4, para aproximadamente $64 \%$ da população, isto é um indicativo de que ocorreu um forte surto da pandemia, uma vez que afetou mais da metade da população.

\section{Conclusão}

O modelo determinístico SIR clássico, Eq. (3.1), mostrou-se adequado quando solucionado pelo método numérico de Runge-Kutta de $3^{\text {a }}$ ordem, pois, ao aplicá-lo a dados reais da Covid-19 no estado do Pará entre os meses de março e abril de 2020 , verificamos uma coerente representação dos infectados por meio de sua solução numérica conforme Fig. 2, pois ela aproxima-se aos dados reais de infectados.

Constatamos que as soluções numéricas da Eq. (3.1), tanto para infectados quanto para recuperados, Fig. 2, apresentam populações crescentes e que estão de acordo com os dados reais obtidos entre os meses de março e abril de 2020, Tab. (1), sendo essas soluções de crescimento exponencial.

Além disso, a partir dessas soluções foi possível realizar previsões referentes ao número de infectados no estado do Pará em dias posteriores ao dia 13 de abril de 2020, Fig. 2. Verificou-se que, em relação ao número de infectados no dia 13 de abril de 2020, Fig. 2, o modelo prevê um crescimento aproximado de infectados de $279 \%$ para o dia 20 de abril de 2020 .

O período de crescimento de infectados, segundo o modelo proposto, está entre os dias04 de maio (dia 65) e 08 de junho (dia 100), Fig. 3. No dia 11 de junho (dia 103), Fig. 3, o número de infectados atinge seu ponto máximo, o que corresponde, aproximadamente, a $11.6 \%$ da população dos municípios de Belém e Ananindeua. A partir do dia 11 de junho de 2020, o número de infectados começa a decrescer, estando assintótico para 
zero a partir do dia 20 de julho (dia 142). Na Fig. 4, os recuperados tendem para, aproximadamente, $64 \%$ da população de Belém e Ananindeua em 20 de julho de 2020, ou seja, mais da metade da população foi infectada pela Covid-19.

Desde que exista um modelo epidemiológico determinístico, é possível aplicar o método numérico de Runge-Kutta de $3^{\mathrm{a}}$ ordem para desenvolver a análise de dinâmica e estimativa de previsão com dados reais de qualquer doença com caráter epidêmico.

A partir da análise do comportamento de epidemias e de suas previsões por modelagem biomatemática, em particular modelagem epidemiológica determinística e/ou estocástica, é possível a entidades governamentais desenvolverem políticas públicas e/ou campanhas de saúde (televisão, rádio e/ou internet) para que não ocorram e/ou para que minimizem epidemias que afligem o mundo.

\section{Referências}

ARAÚJO, A. C. C. Modelação Matemática Epidêmica. 2015. 96 f. Dissertação (Mestrado em Tecnologia Biomédica) - Instituto Politécnico de Bragança, Escola Superior de Tecnologia e Gestão, Bragança/SP, 2015.

BARROSO, L. C.; BARROSO, M. M. A.; FILHO, F. F. C.; CARVALHO, M. L. B.; Maia, M. L. Cálculo Numérico (COM APLICAÇÕES). 2a Ed., Editora Harbra Ltda, São Paulo/SP, 1987.

FCV. FUNDAÇÃO CRISTIANO VARELA. Disponível em: <http://www.fcv.org.br/site/noticia/detalhe/1092>. Acesso em 15/04/2020.

IBGE. INSTITUTO BRASILEIRO DE GEOGRAFIA E ESTATÍSTICA. Disponível em: https://www.ibge.gov. br/cidades-e-estados/pa/ananindeua.html e <https://www.ibge.gov.br/cidades-e-estados/pa/belem.html>. Acesso em: 14/04/2020.

MS. MINISTÉRIO DA SAÚDE. Disponível em: < https://coronavirus.saude.gov.br/>. Acesso em: 14/04/2020. SCHEID, F. Schaum's outline of theory and problems of numerical analysis. $2^{a}$ Ed., McGraw-Hill, New York, 1989.

SESPA. SECRETARIA DE SAÚDE DO ESTADO DO PARÁ. Disponível em: <http://www.saude.pa.gov.br/ coronavirus/>. Acesso em: 14/04/2020.

SOARES, A. L. O. Modelagem Alternativa para Sistemas Epidemiológicos. 2010. 98 f. Dissertação (Mestrado em Matemática Aplicada) - Centro de Matemática, Computação e Cognição/UFABC, Santo André/SP, 2010.

\section{Sobre os autores}

Frayzer Lima de Almeida

Doutorado em Geofísica pela Universidade Federal do Pará (2010). Mestrado em Ciências Geofísicas Geológicas pela Universidade Federal do Pará (2002) Possui bacharelo em Matemática pela Universidade Federal do Pará (2001), e atualmente, é professor adjunto da Universidade Federal do Pará, atuando principalmente nos seguintes temas: geofísica aplicada, métodos elétricos e eletromagnéticos, inversão de dados eletromagnéticos, exploração de petróleo em águas profundas e ultra-profundas.

Recebido em: 16.04.2020

Aceito em: 18.05.2020 